\title{
Otto Frank y Ernest Starling. Más allá de una ley o un mecanismo. Breve reseña histórica
}

\author{
Otto Frank and Ernest Starling. Beyond a law or a mechanism. Brief historical relate
}

\author{
Alejandro Donoso-Fuentes* y Daniela Arriagada-Santis \\ Unidad de Paciente Crítico Pediátrico, Hospital Clínico Dra. Eloísa Diaz I., La Florida, Santiago, Chile
}

\section{Resumen}

La ley o mecanismo de Frank-Starling describe la relación entre la longitud inicial de las fibras miocárdicas y la fuerza generada por su poder de contracción. Aunque ni Otto Frank (1895) como tampoco Ernest Starling (1915) fueron los primeros en descubrir que el volumen diastólico final regula el trabajo del corazón, su participación para este famoso epónimo fisiológico es indiscutible, y de ahí que sus nombres perduraran por más de un siglo en el ambiente de la fisiología, la cardiología y los cuidados intensivos, entre otras disciplinas. Se revisa la biografía de Otto Frank (1865-1944), un excepcional fisiólogo alemán con un amplio conocimiento en física, matemáticas y ciencias naturales, que formuló principios teóricos para la fisiología muscular y cardiovascular, además de muchas otras contribuciones metodológicas e instrumentales. También se examina la vida del gran médico y fisiólogo inglés Ernest Henry Starling (1866-1927), quien elaboró diversos y relevantes aportes científicos, más allá de sus afamadas publicaciones sobre la función circulatoria. Finalmente, el presente artículo comenta en forma breve sus principales y más importantes contribuciones, así como también aspectos menos conocidos de sus logros científicos.

Palabras clave: Fisiología. Corazón. Ley de Frank-Starling. Biografía. Historia de la medicina.

\begin{abstract}
Frank-Starling's law or mechanism describes the relationship between the initial length of myocardial fibers and the force generated by their contraction power. Although neither Otto Frank (1895) nor Ernest Starling (1915) were the first to discover that the final diastolic volume regulates the work of the heart, their participation for this famous physiological eponym is indisputable, enduring their names for more than a century in the environment of physiology, cardiology and intensive care, among other disciplines. The biography of Otto Frank (1865-1944) is reviewed, who was an exceptional German physiologist with extensive knowledge in physics, mathematics and natural sciences who formulated theoretical principles for muscular and cardiovascular physiology, in addition to many other methodological contributions in instrumentals. Also examined the life of the great English physician and physiologist Ernest Henry Starling (1866-1927), who produced various and relevant scientific contributions, beyond his famous publications on circulatory function. Finally, this article briefly comments on its main and most important contributions, as well as less known aspects of its scientific achievements.
\end{abstract}

Keywords: Physiology. Heart. Frank-Starling law. Biography. Medicine history.

\section{Correspondencia:}

${ }^{*}$ Alejandro Donoso-Fuentes

E-mail: adonosofuentes@gmail.com
Disponible en internet: 22-02-2022

Rev Colomb Cardiol. 2022;29(1):16-7

www.rccardiologia.com 0120-5633 / @ 2020 Sociedad Colombiana de Cardiología y Cirugía Cardiovascular. Publicado por Permanyer. Este es un artículo open access bajo la licencia CC BY-NC-ND (http://creativecommons.org/licenses/by-nc-nd/4.0/). 


\section{Introducción}

En más de una oportunidad, en la práctica de reanimación con fluidos, tanto clínicos como investigadores mencionan al rol de la «ley de Frank-Starling» 0 «ley del corazón», la cual establece que el volumen eyectivo del corazón incrementa en respuesta a un aumento del volumen de llenado de este (volumen de fin de diástole), siempre que todos los otros factores permanezcan inalterados ${ }^{1,2}$. Sin embargo, se debe señalar que ni Otto Frank (1865-1944) como tampoco Ernest Henry Starling (1866-1927) fueron sus descubridores $^{3,4}$, mas la participación de ambos en este epónimo es indiscutible, perdurando sus nombres por más de un siglo en las disciplinas de cardio-fisiología ${ }^{5}$, hemodinamia $^{6}$, cardiología ${ }^{7}$, nefrología ${ }^{8}$ y cuidados intensivos $^{9}$, entre otras.

A continuación, se revisa la biografía de Otto Frank, un excepcional fisiólogo alemán con un vasto conocimiento, además, en física, matemáticas y ciencias naturales, lo que le permitió formular principios teóricos en fisiología muscular y cardiovascular, como también muchos otros aportes metodológicos e instrumentales; por otra parte, la del fisiólogo e investigador inglés Ernest Henry Starling, quien elaboró diversos y relevantes aportes más allá de sus afamadas publicaciones sobre la función circulatoria.

Asimismo, se presentan en forma breve sus contribuciones a la ciencia, al igual que aspectos menos conocidos de su prolífica actividad científica. Finalmente, se comenta la época histórica en la cual se desempeñaron, lo que permitirá profundizar nuestro conocimiento en la historia de la medicina.

\section{Friedrich Wilhelm Ferdinand Otto Frank (1865-1944)}

Otto Frank (Fig. 1) nació en 1865 en la localidad de Gross-Umstadt, ubicada al sur del Estado de Hessen. El Dr. Frank realizó sus estudios de medicina en la prestigiosa Universidad de Múnich, finalizándolos en el año 1889. No obstante, esto no fue suficiente para su gran curiosidad intelectual, por lo que decidió completar su formación en ciencias naturales. Así, inmediatamente posterior a su titulación, estudió matemáticas y química en Alemania y Escocia.

En 1890, de retorno a Múnich, trabajó en el laboratorio de Adolf von Baeyer (1835-1917), específicamente en el departamento de química orgánica, al mismo tiempo que aprendía histología en el Instituto de Anatomía bajo la dirección de Karl Kupffer (1829-1902) ${ }^{10}$.

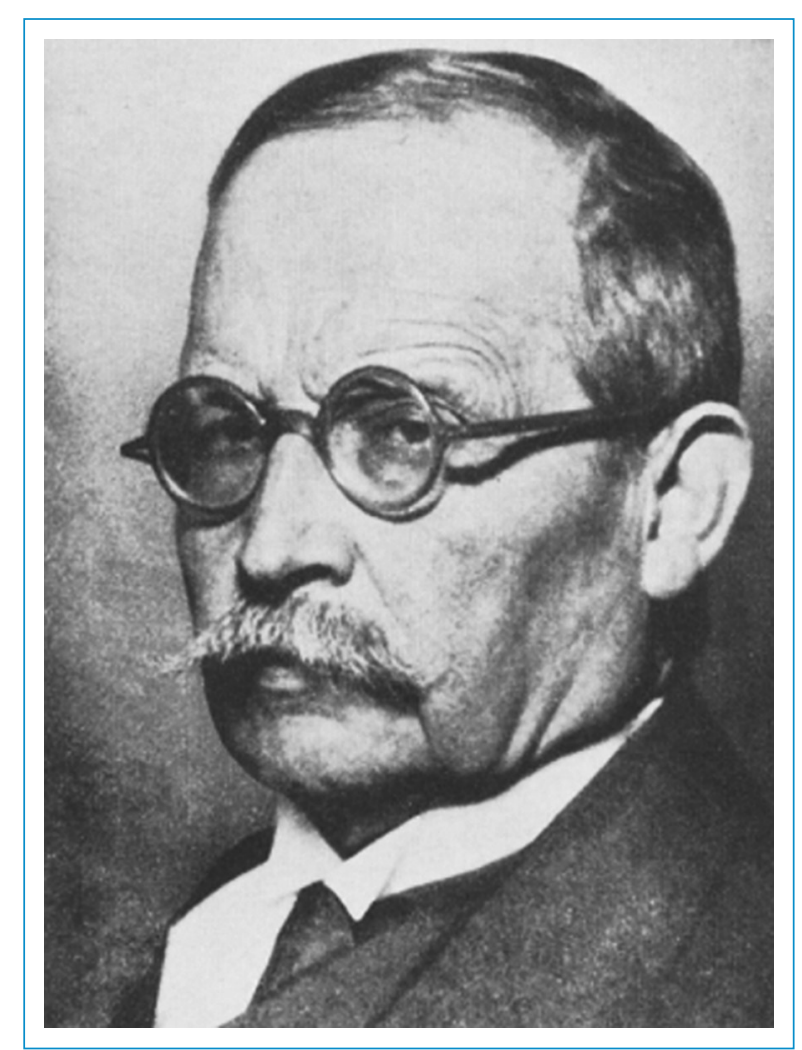

Figura 1. Otto Frank (1865-1944). (Tomada de Wikimedia).

Para el año 1891, Otto Frank se había capacitado en medicina, química, matemáticas, histología, física y zoología, adquiriendo un profundo conocimiento científico, el que le era necesario para poder desarrollar sus modelos animales experimentales. Fue esta sólida formación académica lo que le permitió ser aceptado por Carl Ludwig (1816-1895) en el Instituto Fisiológico de Leipzig, el más afamado centro de investigación europeo de la época ${ }^{11}$, para realizar su tesis doctoral e iniciar así su carrera como fisiólogo. Aquí realizó la mayoría de sus experimentos en 1892 y 1893, siendo su primera tarea asignada la investigación de la absorción intestinal de ácidos grasos ${ }^{12}$. Cabe destacar que, concomitantemente, comenzaba sus estudios experimentales en corazón de rana. No obstante, en 1894 decidió abandonar Leipzig y retornó al Instituto Fisiológico de Múnich como ayudante de Carl von Voit, quien era, a la sazón, jefe de fisiología ${ }^{13}$. Este cambio se originó por la existencia de marcadas discrepancias profesionales con su jefatura, las que se vieron reflejadas en su innovadora y fundamental publicación de 1895 titulada Zur Dynamik des Herzmuskels («Sobre la dinámica del músculo cardíaco») ${ }^{14}$ (vide infra), en la que notoriamente no hizo referencia alguna a sus 
Tabla 1. Temas de investigación y diseños experimentales efectuados por Otto Frank

\begin{tabular}{|c|c|c|}
\hline Tema & Detalle experimental & Principales conclusiones y comentarios \\
\hline Sistema nervioso autonómico & $\begin{array}{l}\text { Modelo animal mamífero (perro) } \\
\text { Estimulación del nervio vago } \\
\text { Estimulación del nervio accelerans }\end{array}$ & $\begin{array}{l}\text { La estimulación vagal ocasionó disminución en la fuerza de } \\
\text { contracción atrial y desarrollo de bradicardia } \\
\text { Predicción del efecto inótropo negativo de la acetilcolina, la } \\
\text { que era desconocida a la fecha } \\
\text { Postuló que ambos nervios influyen en la actividad de los } \\
\text { movimientos cardíacos (aún no se conocían la sinapsis, los } \\
\text { receptores ni los neurotransmisores) }\end{array}$ \\
\hline $\begin{array}{l}\text { Curva presión/volumen y } \\
\text { trabajo cardíaco }\end{array}$ & $\begin{array}{l}\text { En corazón de rana extirpado (no } \\
\text { posee arterias coronarias) } \\
\text { dependiente de precarga y } \\
\text { poscarga }\end{array}$ & $\begin{array}{l}\text { Determinó las curvas isovolumétrica e isotónica máxima, que } \\
\text { cuantificó y graficó } 14,18 \\
\text { Desistió de investigar el mecanismo, se interesó más en los } \\
\text { problemas metodológicos de registro de presión }\end{array}$ \\
\hline $\begin{array}{l}\text { Efectos de preparados } \\
\text { digitálicos sobre el corazón }\end{array}$ & En corazón de rana extirpado & Detalló sus efectos sobre el inotropismo y el cronotropismo \\
\hline Fuerzas del sarcómero & En corazón de rana extirpado & $\begin{array}{l}\text { Estableció paralelos entre el músculo esquelético y el } \\
\text { músculo cardíaco. Sugirió que el diagrama de presión- } \\
\text { volumen del corazón de la rana coincide con el diagrama de } \\
\text { tensión-longitud del músculo esquelético } \\
\text { Describió la contracción isométrica e isotónica del corazón } \\
\text { Postuló que los resultados centrales de los experimentos } \\
\text { realizados con todo el órgano (corazón de rana) también se } \\
\text { pueden transferir a los elementos musculares a un nivel } \\
\text { microscópico. No usó el término sarcómero, sino que más } \\
\text { bien se refirió a la estriación cruzada } \\
\text { Visualizó las funciones de estructuras, como la interacción de } \\
\text { actina y miosina (fuerza contráctil) y la fuerza elástica de la } \\
\text { titina, la que se descubrió ocho décadas después }\end{array}$ \\
\hline $\begin{array}{l}\text { Entendimiento y mejora de la } \\
\text { instrumentación }\end{array}$ & & $\begin{array}{l}\text { Desarrolló y perfeccionó dispositivos para mejorar la precisión } \\
\text { de las mediciones } \\
\text { Detalló la dinámica de la transmisión y la atenuación de la señal } \\
\text { Describió un dispositivo con base en lámparas y lentes que } \\
\text { registraba fotográficamente los movimientos }\end{array}$ \\
\hline $\begin{array}{l}\text { Experimentación con } \\
\text { animales de sangre caliente }\end{array}$ & $\begin{array}{l}\text { Múltiples preparaciones con } \\
\text { mamíferos (perros, gatos, cobayas } \\
\text { y erizos de tierra) }\end{array}$ & $\begin{array}{l}\text { Confirmó factores de los cuales depende el volumen eyectivo } \\
\text { No presentó curvas de presión/volumen }\end{array}$ \\
\hline
\end{tabular}

trabajos previos e incluso efectuó importantes críticas metodológicas al diseño en el que había participado ${ }^{3}$.

Esta singular publicación proporcionó una visión del corazón desde el punto de vista de la mecánica muscular esquelética, lo que se corroboró en sus propias palabras, en las que señaló: «la idea básica que guio mi investigación fue que el conocimiento del rendimiento mecánico del músculo cardíaco debería relacionarse, en la medida de lo posible, con lo que ya se sabe sobre el rendimiento mecánico del músculo esqueléti$\operatorname{co}^{15}$. Durante su vida, Frank realizó 133 publicaciones sobre muy diversos temas (Tabla 1$)^{7}$.

Finalmente, es importante señalar que Otto Frank estudió el concepto básico de pulso arterial como también la oscilación periódica de la onda de presión y su propagación desde el corazón a los órganos ${ }^{16}$. De esta manera, evaluó de manera global la fisiología vascular, enfocándose en las curvas de la presión arterial. Así, hace más de un siglo logró describir importantes fenómenos y parámetros que, en la actualidad, son las bases del análisis de la onda de pulso ${ }^{6}$.

Según sus biógrafos, Otto Frank era un hombre de carácter difícil y muy crítico respecto a la incorporación de innovaciones y reformas. No apreciaba a las mujeres en medicina, los estudiantes le temían en los exámenes y bloqueó la fundación de un instituto bioquímico, el establecimiento de una clínica pediátrica y un instituto de historia de la medicina en la Universidad de Múnich ${ }^{17}$.

Hacia 1908 asumió como sucesor de Carl von Voit en Múnich, siendo esta una difícil decisión ya que le privaba de una considerable cantidad de tiempo y energía, la que deseaba usar como investigador. Empero, permaneció leal a Múnich ${ }^{10}$ hasta que, 
finalmente, debido a su oposición al régimen nacionalsocialista (lo que se reflejó tanto en sus conferencias para estudiantes como en su vida pública), fue exonerado de su cargo en el año 1934, viéndose obligado a aceptar el estatus de emérito. Otto Frank falleció en Múnich en el mes de noviembre de 1944. A los 2 meses, su laboratorio fue bombardeado.

\section{Diseño experimental clásico y sus precedentes}

En su preparación de corazón aislado de rana, donde la circulación estaba abierta y, por ende, el llenado ventricular (precarga) podía ser regulado en forma independiente de la poscarga, su principal contribución fue que observó que, ante un incremento del volumen de fin de diástole, la presión isométrica ventricular aumentaba, resultando ser dependiente del llenado inicial; sin embargo, si se sobrepasaba cierto nivel de llenado, la presión declinaba. Además, ante un mayor volumen de llenado diastólico la velocidad máxima de ascenso de presión $\left(d P / d t_{\text {max }}\right)$ se incrementaba ${ }^{14}$. Finalmente, compiló todos los datos en el diagrama de presión-volumen, permitiéndole el cálculo del trabajo cardíaco ${ }^{18}$.

No obstante, el fisiólogo Johann Nepomuk Czermak (1828-1873) había realizado una versión simplificada de este modelo experimental durante su demostración de 1872 en el spectatorium de Leipzig ${ }^{19}$. Además, otro estudio similar, destinado a conocer el efecto de las presiones de llenado en la contractilidad cardíaca, había sido efectuado casi 30 años antes por tres jóvenes investigadores del Instituto Fisiológico Carl Ludwig de la Universidad de Leipzig, llamados E. Cyon, J. Coats y H. Bowditch ${ }^{3}$.

Se debe señalar que el mayor interés y preocupación de Otto Frank estaba puesto sobre los problemas metodológicos, como la construcción de manómetros («uno simplemente [no puede] penetrar en los misterios de la naturaleza sin palancas y tornillos") y el cuidadoso análisis matemático de las curvas de presión registradas del sistema cardiovascular?

Posteriormente, en las primeras dos décadas del siglo xx, el gran debate fisiológico continuó. Hermann Straub (1882-1938), en la primera Clínica Médica en Múnich, hizo una importante contribución en 1914 con su tesis posdoctoral sobre el tema Dinámica del corazón de mamífero ${ }^{6}$, y en 1915, el fisiólogo italiano Dario Maestrini (1886-1975) publicó su Legge del cuore con similares conclusiones tras finalizar sus experimentos en corazones de ranas.

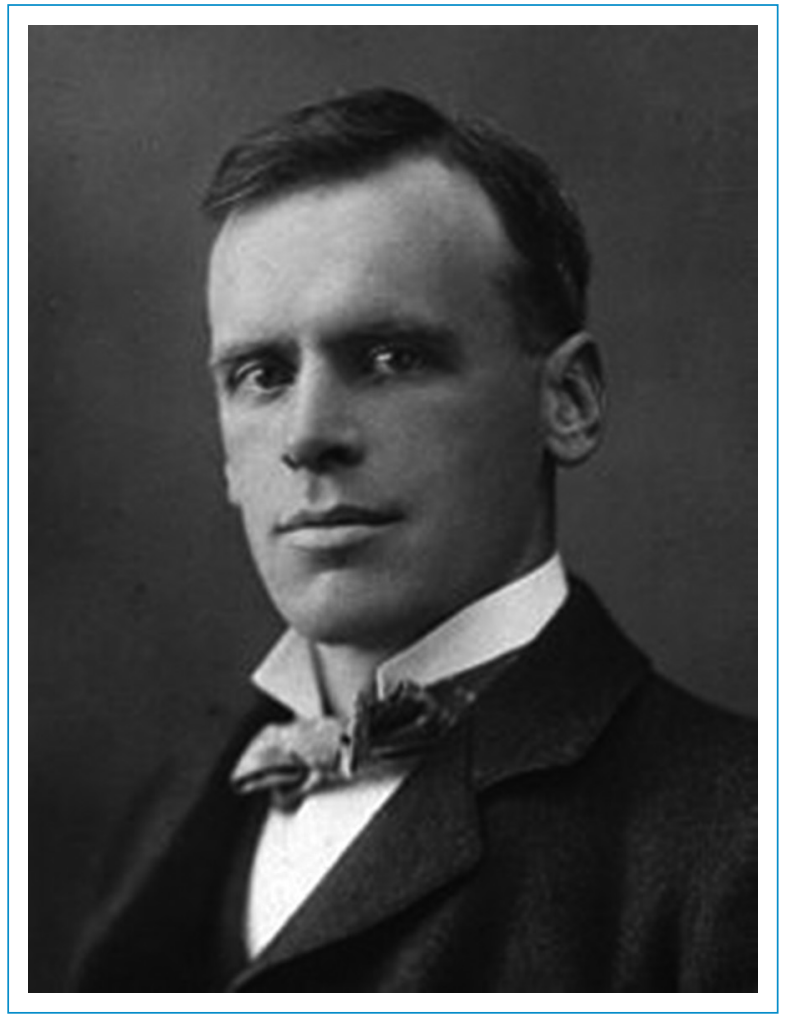

Figura 2. Ernest H. Starling (1866-1927). (Tomada de Historia de la Medicina [NLM], vía Wikimedia).

Finalmente, 20 años después de la comunicación de Frank, el fisiólogo inglés Ernest $\mathrm{H}$. Starling confirmó estos hallazgos en su preparación cardiopulmonar canina (vide infra) ${ }^{20}$.

\section{Ernest Henry Starling (1866-1927)}

Ernest H. Starling (Fig. 2) nació en 1866 en Londres y fue el primogénito de siete hijos dentro de una familia de escasos recursos económicos y con una profunda convicción religiosa cristiana. Su padre era abogado de la corona británica, quién se desempeñaba en Bombay y regresaba cada 3 años para visitar a la familia. Por ende, la crianza durante su infancia estuvo a cargo de su madre y, en ocasiones, el mismo Ernest debía asumir el rol paterno en la familia. En 1882, a los 16 años, inició sus estudios en la escuela de medicina del Guy's Hospital, donde logró un rendimiento académico extraordinario, graduándose en 1889. El año siguiente, Starling publicó su primer artículo científico junto con su compañero Frederick Gowland Hopkins, quien sería el futuro Premio Nobel de Fisiología y Medicina de 1929 por el descubrimiento del rol de las vitaminas ${ }^{21}$. 
Una huella imborrable durante su formación médica fue el haber concurrido durante el verano de 1885 al laboratorio del fisiólogo alemán Wilhem Kühne (18371900) en la Universidad de Heidelberg, donde investigaba el proceso químico de la digestión y fue quien acuñó el término bioquímico «enzima»"21.

Durante gran parte de su vida profesional, Starling trabajó en la facultad del University College of London, conjuntamente con William Bayliss (1860-1924). Se señala que Bayliss presentaba características personales de ser alguien metódico y cauteloso, mientras que Starling era descrito como un hombre de personalidad optimista y con un fuerte encanto personal, pero a la vez controversial, falto de diplomacia, impaciente y poco prudente ${ }^{22}$. En su primer trabajo conjunto, describieron el exitoso registro electrocardiográfico de un mamífero, demostrando que la contracción se iniciaba en la base y continuaba hacia el ápex ${ }^{23}$. Posteriormente, sus publicaciones científicas conjuntas durarían por 15 años, algo no muy habitual en Starling, pues frecuentemente establecía coautorías en no más de una o dos y, excepcionalmente, tres oportunidades.

En 1892, Starling realizó estancias formativas con Rudolf Heidenhain (1834-1897), quien trabajaba en el estudio de la linfa, y con lliá Méchnikov (1845-1916) en el Instituto Pasteur de París. De regreso a Londres, abordó los temas de la producción de linfa ${ }^{24}$, de la permeabilidad capilar, así como del efecto fisiológico de las fuerzas osmóticas, lo cual le permitió elaborar lo que actualmente se conoce como "equilibrio de Starling", logrando así una explicación cuantitativa del transporte transcapilar de fluidos (1896).

En 1899, Starling y Bayliss formularon la «ley del intestino" al explicar el control nervioso del peristaltismo. Posteriormente, durante sus estudios de la secreción pancreática, descubrieron la secretina $(1902)^{25}$, para finalmente Starling, en 1905, acuñar el término «hormona» durante la conferencia Croonian en el Royal College of Physicians, la cual tituló La correlación química de las funciones del cuerpo ${ }^{26}$. Esta idea contribuyó, en forma definitiva, al desarrollo de la concepción moderna de la endocrinología. El concepto de regulación hormonal de las glándulas digestivas remeció las teorías imperantes de la época, especialmente las propagadas por Ivan Pavlovi27.

Por lo ya mencionado, se puede apreciar que la fisiología cardíaca no era aún un tema relevante en la investigación de Starling. La serie de experimentos que dieron formulación a la «ley del corazón» comenzó en $1912^{28}$ y concluyó casi un decenio después ${ }^{29}$. En 1915 dio su célebre Conferencia Linacre en la Universidad de Cambridge (vide infra), la cual fue publicada 3 años después ${ }^{29}$. No obstante, este concepto fisiológico fundamental corresponde a la convergencia de las observaciones iniciales de un grupo excepcional de fisiólogos europeos y estadounidenses ${ }^{4,15}$.

Su permanente actividad científica se consolidó con la publicación del clásico texto de fisiología en 19124; empero, esta se vio interrumpida con el estallido de la Gran Guerra, conflicto en el que decidió participar activamente, llegando a ocupar el cargo de director de investigación en Millbank Laboratories, donde experimentó en métodos para contrarrestar el efecto de gases tóxicos. Posteriormente, fue enviado como teniente coronel a Tesalónica, donde prontamente entabló controversias con las autoridades, regresando a Londres como civil. Paradójicamente, el único premio de carácter no académico que recibió en vida Starling fue por su servicio en Tesalónica ${ }^{22}$.

Una de las razones que motivaron la rápida aceptación por la comunidad científica de los postulados de Starling fue la guerra, pues esta logró unificar la fisiología y la medicina británica, que se encontraba impedida de recibir la gran influencia de los programas docentes alemanes, por aquella época líderes mundiales en la medicina científica ${ }^{15}$.

En 1922, como profesor de la Royal Society, se dedicó a estudiar la fisiología renal, describiendo un par de años después la reabsorción tubular de agua. El último trabajo publicado por Starling fue en conjunto con M. Visscher sobre fisiología cardíaca ${ }^{30}$. Una década después, T. Harrison usaba las observaciones de Starling para explicar el síndrome clínico de falla cardíaca ${ }^{31}$.

Starling tuvo una relación profesional muy cercana con al menos siete premios Nobel durante su vida; sin embargo, a pesar de haber sido nominado en tres ocasiones, jamás obtuvo el afamado galardón ${ }^{22,32}$, presentando una visión crítica y despectiva sobre este reconocimiento ${ }^{33}$. Además, nunca recibió el título de caballero, por su crítica, a menudo abierta, al escaso entendimiento por parte de las autoridades gubernamentales de la cien$\mathrm{cia}^{34}$, como también por haber sido uno de los primeros en el período de posguerra en reanudar su colaboración cientíica con los fisiólogos alemanes.

En sus últimos años de vida, su salud se vio gravemente comprometida por un cáncer de colon que requirió hemicolectomía y dejó como secuelas claudicación de su extremidad ${ }^{22}$. Ernest Starling dio su última conferencia (A century of physiology) en Londres en el mes de febrero de 1927 y murió 3 meses después debido a una insuficiencia cardíaca. Se encuentra sepultado en Kingston, Jamaica. 


\section{Diseño experimental clásico para la determinación del gasto cardíaco}

Starling comenzó sus trabajos en el corazón de mamíferos con el objetivo de explicar por qué el gasto cardíaco permanecía constante sobre un rango bastante amplio de presión arterial, frecuencia cardíaca y temperatura ${ }^{35}$.

En 1914, junto con Patterson, utilizó su preparación aislada de corazón-pulmón (en perros anestesiados y ventilados a presión positiva) para estudiar la relación entre afluencia venosa, presión venosa y gasto cardíaco. Para esto, mantuvo intactas la circulación pulmonar y coronaria, y ligó la vena cava inferior, la aorta distal y las ramas del arco aórtico. Una cánula en el arco aórtico desviaba el flujo sistémico hacia un circuito extracorpóreo, bombeando el ventrículo izquierdo sangre a través del mismo hacia un reservorio sanguíneo elevado. Por gravedad, se extraía la sangre desde este a través de una cánula que conducía a la vena cava superior, y mediante una abrazadera en dicha cánula (resistencia) se regulaba la velocidad del flujo sanguíneo hacia la aurícula derecha. Esto le permitió demostrar que, en un amplio rango, el corazón era capaz de expulsar el volumen de sangre que el sistema retornaba a la aurícula. Dado que el flujo hacia la aurícula derecha coincidía con el flujo desde la aorta, Starling denominó a ambos como "gasto cardíaco»20,36.

Al abrir la resistencia, la sangre fluía a un ritmo creciente desde el reservorio venoso hacia la aurícula derecha. Inicialmente, la presión auricular aumentaba de manera lenta, aunque existía un límite en la capacidad del corazón para adecuar el aumento de la tasa de retorno de sangre. Más allá de este límite, el corazón (en sus palabras) «se fatigó». Starling graficó la presión auricular derecha en el eje $y$ (ordenada) y el gasto cardíaco en el eje $x$ (abscisa), siendo algo confuso en la actualidad, pues ninguna de estas dos variables era independiente en su experimento ${ }^{20}$. En reproducciones posteriores, al representarla se ubicó la presión auricular derecha (presión venosa central) en la abscisa, motivo por el que se asume erróneamente que esta es una variable independiente ${ }^{36}$. Sin embargo, el texto de Starling no sugiere que la presión auricular derecha sea una variable independiente que controle el volumen sistólico o el gasto cardíaco. La variable realmente independiente era la magnitud de apertura de la válvula de resistencia del flujo hacia la aurícula derecha. Starling enfatizó que la presión auricular derecha aumentaba como consecuencia del incremento del retorno sanguíneo al corazón. Este hallazgo significaba que el corazón podría acomodar cantidades variables del retorno venoso hasta un límite fisiológico. Así, a medida que aumentaba el retorno sanguíneo más allá del límite de acomodación, la sangre se acumulaba en el corazón y el gasto cardíaco disminuía ${ }^{36}$.

De esta manera, cabe destacar que la curva de Starling no implica que el alza de la presión auricular derecha aumente el gasto cardíaco ${ }^{36}$. En algunos de sus experimentos, Starling incrementó la resistencia al flujo de salida aórtico ocasionando un aumento de la presión auricular derecha, pero no del gasto cardíaco ${ }^{20}$. Se ha señalado que Starling nunca compartió un gran entusiasmo por su curva, pues no la mencionó en su famosa Conferencia Linacre sobre el corazón (1915), en la cual integró sus experimentos ${ }^{37}$, como tampoco en su libro de fisiología médica. Para Starling, la curva era solo parte de los fundamentos para una visión más importante: la «ley del corazón», la que fue publicada en $1918^{29}$ y que señala que «la energía total liberada en cada latido está determinada por el volumen diastólico del corazón y, por lo tanto, por el largo de la fibra al comienzo de la contracción " ${ }^{30}$. En pocas palabras, el trabajo miocárdico variaba con la longitud inicial de la fibra del miocito.

\section{Conclusiones}

Otto Frank fue uno de los pioneros de la fisiología a fines del siglo xix, dedicándose a la búsqueda de los fundamentos matemáticos, metodológicos y teóricos para una comprensión cabal del complejo rendimiento del corazón y del sistema circulatorio. Es notable cómo sus publicaciones, realizadas hace más de un siglo, muestran una riqueza de supuestos y proyecciones teóricas referentes al sarcómero, desarrollo de modelos de contracción, termodinamia muscular, análisis de la curva presión/volumen y diversos problemas metodológicos ${ }^{7}$. Muchos de estos conceptos enunciados fueron validados experimentalmente y morfológicamente en las décadas siguientes. En la actualidad, su investigación conserva plena vigencia, lo que se confirma en los diversos modelamientos matemáticos hemodinámicos y de simulación cardiovascular para el estudio fisiopatológico, en particular de la falla cardíaca, y el uso de diversas intervenciones terapéuticas destinadas a su tratamiento ${ }^{38}$.

Los méritos científicos de Ernest Starling son ampliamente conocidos y recordados en el ámbito de la cardiología y la fisiología circulatoria, siendo la formulación de la «ley del corazón» (la que lleva su nombre) y el «equilibrio de Starling» sus principales aportes ${ }^{22,39}$. No obstante, en otras disciplinas médicas, como la 
endocrinología (con la incorporación del término «hormona" y el descubrimiento de la secretina) y la nefrología, su participación es menos conocida. Sus numerosas publicaciones científicas, que combinan fisiología con medicina clínica, contribuyeron sustancialmente a que la fisiología se transformara de una disciplina cualitativa a una ciencia cuantitativa, permitiendo de este modo profundizar el conocimiento en fisiopatología básica y el diagnóstico clínico ${ }^{22}$.

Existe evidencia de que el rol del volumen de fin de diástole en el funcionamiento cardíaco era ampliamente conocido por los fisiólogos durante la segunda mitad del siglo XIX, décadas antes de que Starling describiera la «ley del corazón». A pesar de ello, la curva originada de su experimento, luego de más de un siglo de investigación, permanece válida y es esencial en el entendimiento de la fisiología circulatoria.

Parece apropiado, con ocasión de los 125 años de la descripción de Otto Frank, recordar la historia detrás de estos dos hombres, cuyos logros científicos han resistido la prueba del tiempo y los cuales estarán indisolublemente ligados en la historia de la medicina y la fisiología.

\section{Financiamiento}

Los autores declaran que no hay ningún tipo de financiamiento para este artículo.

\section{Conflicto de intereses}

Los autores declaran no tener conflicto de intereses alguno.

\section{Responsabilidades éticas}

Protección de personas y animales. Los autores declaran que para esta investigación no se han realizado experimentos en seres humanos ni en animales.

Confidencialidad de los datos. Los autores declaran que en este artículo no aparecen datos de pacientes.

Derecho a la privacidad y consentimiento informado. Los autores declaran que en este artículo no aparecen datos de pacientes.

\section{Bibliografía}

1. Holubarsch C, Ruf T, Goldstein DJ, Ashton RC, Nickl W, Pieske B, et al. Existence of the Frank-Starling mechanism in the failing human heart. Investigations on the organ, tissue, and sarcomere levels. Circulation. 1996;94:683-9.
2. Westerhof N, Stergiopulos N, Noble MIM, Westerhof B. Snapshots of hemodynamics. $2^{\text {nd }}$ ed. New York: Springer; 2010.

3. Zimmer HG. Who discovered the Frank-Starling mechanism? News Physiol Sci. 2002;17:181-4.

4. Guz A. Chairman's Introduction. En: Ciba Foundation Symposium 24. The Physiological Basis of the Starling's Law of the Heart. Amsterdam: Elsevier, Excerpta Medica; 1974. p 1-5.

5. Sequeira V, van der Velden J. Historical perspective on heart function: the Frank-Starling Law. Biophys Rev. 2015;7:421-47.

6. Middeke M. The pioneer in hemodynamics and pulse-wave analysis, Otto Frank. J Am Soc Hypertens. 2016;10:290-6.

7. Kuhtz-Buschbeck JP, Drake-Holland A, Noble MIM, Lohff B, Schaefer J. Rediscovery of Otto Frank's contribution to science. J Mol Cell Cardiol. 2018;119:96-103. [Published correction appears in J Mol Cell Cardiol. 2018;123:58.]

8. Middeke M. Otto Frank, der Dynamiker. Dtsch Med Wochenschr. 2012;137:2706-11.

9. De la Oliva P, Menéndez-Suso JJ, Iglesias-Bouzas M, Álvarez-Rojas E, González-Gómez JM, Roselló P, et al. Cardiac preload responsiveness in children with cardiovascular dysfunction or dilated cardiomyopathy: a multicenter observational study. Pediatr Crit Care Med. 2015;16:45-53.

10. Wezler K. Otto Frank zum Gedächtnis [In memoriam Otto Frank]. Z Biol. 1950;103:91-122.

11. Zimmer HG. Carl Ludwig: the man, his time, his influence. Pflugers Arch. 1996;432(3 Suppl):R9-22.

12. Frank O. Die Resorption der Fettsäuren der Nahrungsfette mit Umgehung des Brustganges [Resorption of nutritional fatty acids circumventing the thoracic duct]. Arch Anat Physiol. 1892;497-512.

13. Zimmer HG. Otto Frank and the fascination of high-tech cardiac physiology. Clin Cardiol. 2004;27:665-6.

14. Frank O. Zur Dynamik des Herzmuskels. Z Biol. 1895;32:370-437.

15. Katz AM. Ernest Henry Starling, his predecessors, and the "Law of the Heart". Circulation. 2002;106:2986-92.

16. Frank O. Der Puls in den Arterien. Z Biol. 1904;46:441-53.

17. Wildi-Torster A. Otto Frank und das Werden der Physiologie in München. Schriftenreihe der Münchener Vereinigung für Geschichte der Medizin e.V., vol. 24, Verlag UNI-DRUCK; 1997.

18. Frank O. Die Grundform des Arteriellen Pulses. Erste Abhandlung. Mathematische Analyse. Z Biol. 1898;37:483-526.

19. Zimmer HG. Johann Nepomuk Czermak and his isolated frog heart. Clin Cardiol. 2005;28:257-8.

20. Patterson SW, Starling EH. On the mechanical factors which determine the output of the ventricles. J Physiol. 1914;48:357-79.

21. Fye WB. Ernest Henry Starling, his law and its growing significance in the practice of medicine. Circulation. 1983;68:1145-8.

22. Henriksen JH. Ernest Henry Starling (1866-1927): the scientist and the man. J Med Biogr. 2005;13:22-30.

23. Bayliss WM, Starling EH. Report on the electromotive phenomena of the mammalian heart. Br Med J. 1891;2:186-7.

24. Fine LG. Ernest Henry Starling (1866-1927) on the formation and reabsorption of lymph. Nephron Physiol. 2014;126:9-17.

25. Bayliss WM, Starling EH. On the causation of the so-called 'peripheral reflex secretion' of the pancreas. Proc Ruy Soc B. 1902;69:352-3.

26. Starling EH. Croonian Lecture. On the chemical correlation of the functions of the body. Lancet. 1905;2:339-41.

27. Babkin BP. Pavlov. A biography. Chicago: University of Chicago Press; 1949

28. Knowlton FP, Starling EH. The influence of variations in temperature and blood pressure on the performance of the isolated mammalian heart. J Physiol. 1912;44:206-19.

29. Starling $\mathrm{EH}$. The Linacre Lecture on the Law of the Heart. London: Longmans, Green and Co.; 1918.

30. Starling $E H$, Visscher MB. The regulation of the energy output of the heart. J Physiol. 1927;62:243-61.

31. Harrison TR. Failure of the circulation. Baltimore: Williams \& Wilkins; 1936.

32. Henriksen JH. Starling, his contemporaries and the Nobel Prize. One hundred years with hormones. Scand J Clin Lab Invest Suppl. 2003;238:1-59.

33. Starling EH. Discovery and research. Nature. 1924;113:606-7.

34. Starling EH. Natural science in education: notes on the report of the Committee on the Position of Natural Science in the Educational System of Great Britain. Lancet. 1918;ii:365-6.

35. Markwalder J, Starling EH. On the constancy of the systolic output under varying conditions. J Physiol (Lond). 1914;48:348-56.

36. Berlin DA, Bakker J. Starling curves and central venous pressure. Crit Care. 2015;19:55.

37. Starling EH. The Linacre lecture on the law of the heart given at Cambridge, 1915. Nature. 1918;101:18.

38. Doshi D, Burkhoff D. Cardiovascular simulation of heart failure pathophysiology and therapeutics. J Card Fail. 2016;22:303-11.

39. Fye WB. Ernest Henry Starling. Clin Cardiol. 2006;29:181-2. 